\title{
Practical guidance for early career researchers dealing with tardy or unresponsive co-authors
}

\author{
Steven J. Cooke, Michael R. Donaldson, and Timothy D. Clark \\ Steven J. Cooke (Steven_Cooke@carleton.ca), Fish Ecology and Conservation Physiology Laboratory, Institute of \\ Environmental Science and Department of Biology, Carleton University, Ottawa, ON, Canada K1S 5B6
}

Michael R. Donaldson, Department of Natural Resources and Environmental Sciences, University of Illinois at Urbana, Urbana, IL, USA 61820

Timothy D. Clark, Australian Institute of Marine Science, Townsville, QLD, Australia 4810

Everyone that has experience in academic publishing is all too familiar with the "tardy co-author"-or worseone that is completely unresponsive. As an author, one works tirelessly in an attempt to improve a paper and move it one step closer to submission to a peer-reviewed outlet only to have it stall (sometimes permanently) on the desk of a co-author. This would seem to be particularly problematic for early career researchers (such as students, post docs and pre-tenure professors/lecturers) where there is a veritable "requirement" to share one's work with the broader scientific community via peerreviewed publications (Schäfer et al. 2011). Undue delays in an already rather slow process (i.e., peer review and publishing; Rowland 2002) could impede one's ability to secure scholarships, awards, or research funding, make one less competitive for employment or further studies, or perhaps worst, lead to the work being abandoned to never see the light of day. This last outcome not only hurts the author but also the knowledge receptor community by failing to deliver on the promise of disseminating research that is often funded by tax payers or donors. This issue is magnified when research involves human or animal subjects or endangered organisms.

What is an author to do to move a paper forward when dealing with a tardy or unresponsive co-author? All three of us have had to deal with this situation on multiple occasions - sometimes with desirable outcomes and sometimes with undesirable ones. We have always attempted to seek guidance on which actions to take but have failed to find help in written form; hence, the basis for this paper. We admit from the start that there is no "correct" answer nor a singular path to follow. Nonetheless, we feel that it is prudent to share our collective experiences, directed largely towards early career researchers, to provide them with strategies for helping to move a paper one step closer to journal submission (Figure 1). We acknowledge that the path forward is context dependent. If there is only one coauthor (and it is a supervisor), the path is inherently more difficult than if it is for a multi-author paper (say six co-authors) and only one of the contributors is unresponsive.

Although it is not possible to always know why a coauthor is being tardy or entirely disengaged, there are some obvious reasons - some we have heard and others we have inferred. The most obvious one is simply time - "I am too busy right now." Such a response is certainly fair, as early career researchers often recognise later in life when they find themselves juggling an overwhelming number of tasks that they never thought possible. However, the response should be followed up with a reasonable timeline and it would seem ignorant to say things like "maybe next year" or "this is my teaching term" or "I am on sabbatical." The problem arises when promised deadlines are agreed upon and repeatedly missed. Another possible reason is that the person forgot or failed to make it a priority such that it 


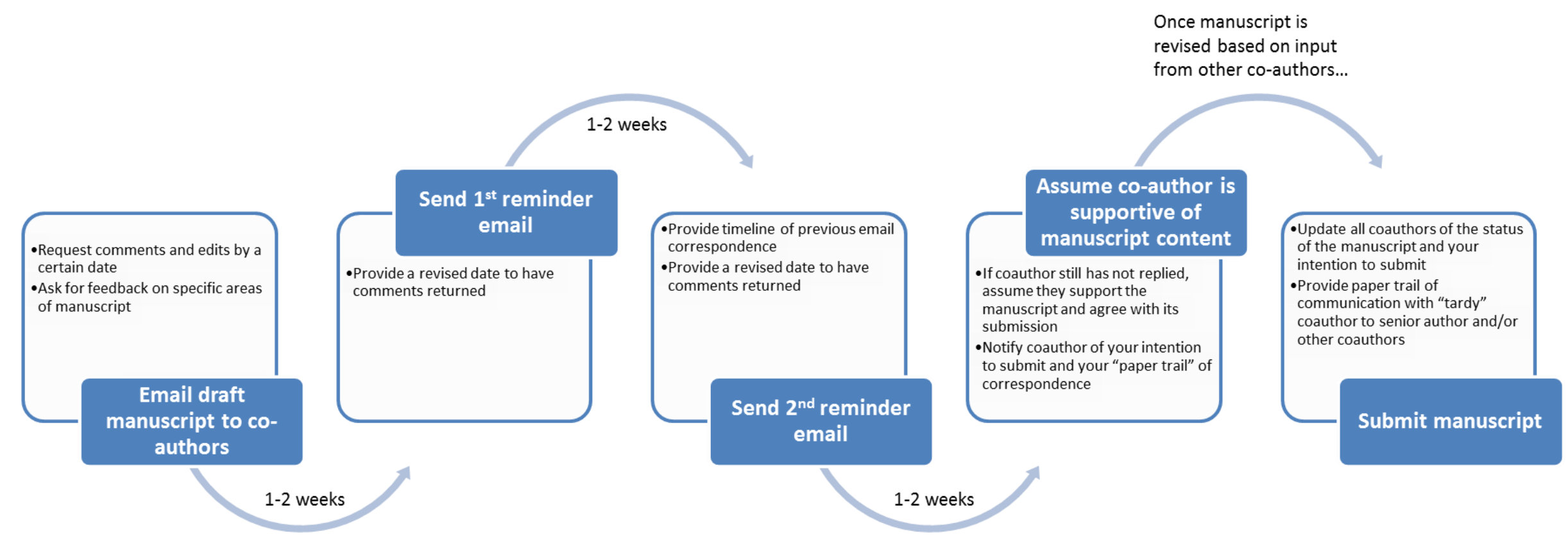

Figure 1. A flow chart showing an example sequence of events for working with a tardy or non-responsive co-author when preparing a manuscript for submission to a journal. 
has been shuffled down the "to do" list. This has happened to all of us - both serving as authors and "that" forgetful co-author. A simple email like "Hey so and so - Just wondering if you had a chance to look at my paper yet? I am really keen for your input. I attach it again here for your reference. I was wondering when I might expect to receive your input?" serves to both remind people that may have forgotten and to push it up higher on the priority list. We have encountered some instances where a paper has apparently sat with a coauthor for weeks yet they did not see it come into their inbox, due either to user or technical error. This is all to say that moving a paper forward is also as much the responsibility of the lead author as it is the tardy coauthor. One cannot simply assume that prolonged silence after sending along a paper for comment is the co-author being slow; they may simply be unaware that they have a task to do. We suggest that if there has not been acknowledgement from a co-author after 1-2 weeks that they have received the paper to review, it is appropriate to send a gentle reminder.

Our position is rather simple. We consider that dissemination of research to the broader scientific community via peer-reviewed outlets is not only part of the research process, but perhaps the ultimate reason for conducting research in the first place. In our view, there is an expectation and responsibility to share one's work with the broader community. Therefore, we present a series of steps intended to help move work forward. If the co-author is not the supervisor and there is another "senior" person involved that can help to mediate, that is often desirable. The reality is that it is difficult to write a paper by committee; co-authors are co-authors for a variety of reasons, which may not require any formal role in writing but rather general editing of the manuscript. If the paper has more than two authors it is often desirable to identify a lead author along with a supporting person (we usually consider that the senior author assumes this role). In that sense, the goal is for those two individuals to generate a paper that is as close to final as possible. Letting other co-authors know that the paper is ready to move forward in the eyes of both the lead senior authors will often help to elicit a rapid response. Consider the alternative: being asked to review a paper, especially by a novice writer that is in an early draft form and excessively long. Polishing a paper is often less burdensome than dealing with early drafts. The idea of course is not to use deceit to advance a paper because even a superficially polished paper may suffer from a variety of fatal flaws. The point is that if the reason a tardy co-author is not doing the review is because they are overwhelmed by the thought of starting the process, a gentle nudge such as this may be sufficient to start the process (similar to a Jedi mind trick!). One may request high-level general comments from one co-author and more detailed technical and editorial comments from another on a specific part of the manuscript (e.g., "I am particularly keen on your input on my interpretation of $X$ part of the paper").

No doubt the most frustrating type of co-author is the one that is completely unresponsive. After the usual chain of emails (do keep time-stamped records by cc'ing yourself on email correspondence), it may be appropriate to have another co-author (e.g., senior author) attempt contact. It can also be prudent for you or the co-author to try alternative communication means. For example, attempt to schedule a face-to-face meeting, knock on their door, or pick up the phone. It is easy to ignore emails but not as easy to ignore more intimate means of communication. If that fails, it may be necessary to investigate with colleagues whether something has happened that makes it difficult for the author to communicate. Examples could include a death in a family, research or conferences in a remote location, health or personal problems. In such situations, tact is important. At the same time, one must be reasonable in that not everything can be halted indefinitely. We consider a several month delay to be reasonable provided that the individual has committed to a timeline. However, if there is no response to at least three email queries by the lead author, one by another author (if possible), and attempts to contact the co-author via other means, it is reasonable to send a final communication stating the following... "I intend to submit the MS to X outlet on X date. I am still keen for your input but I can only presume that the silence indicates that you are in agreement with the content of the manuscript. I have attempted to contact you $X$ times on $X$ dates. I intend to submit the paper in its present form to $X$ outlet on $X$ date with you as a co-author unless I hear from you otherwise. If you are able to provide comments at a later date I will incorporate them during the revision phase..." As eluded to above, this tactic requires one to maintain a "paper trail" of communication to support decisions if ever queried by academic officials, professional bodies or a publisher/editor. Journals require a statement that all authors agree to a submission so such a paper trail (i.e., where failure to reply is taken as tacit approval) is needed. The key is to escalate slowly, being cognisant of holidays or religious periods that may inherently require more time. Depending on the circumstances, the alternative-albeit more severeapproach is to consult with your other co-authors and determine whether or not an unresponsive (or exceptionally tardy) individual should be removed from the manuscript's authorship. That topic is beyond the scope of this article but we refer readers to the editorial policies of various journals and the Committee on Publication Ethics (COPE; see www.publication ethics.org).

We also acknowledge that the problem is magnified further if the tardy co-author is in a position of power 
relative to the author (e.g., supervisor, mentor, employer) such that anything that resembled "pressure" to move the paper forward could lead the co-author to take offence. If such an authority figure is unresponsive it may be worthwhile to seek assistance from a different co-author of equal or higher status with the tardy one or as a last resort contact a departmental chair, head or director.

As authors advance in their careers and gain more experience with different collaborators, it may be possible to avoid working with tardy or unresponsive coauthors (this obviously depends on the track record of tardiness/unresponsiveness). Supervisors can protect their students from such situations by not putting them in a position where there is a known unresponsive author involved. Collaborations - the good ones-are positive and even fun and it is entirely appropriate to choose whom to avoid. At some level it is about mutual respect and consideration for each other's best interests. If one takes the time to generate a paper then is it not only fair that a co-author comments in a timely manner? Admittedly, all of us enjoy and have a passion for writing and editing. For us it is simply impossible not to open a file and begin to read and think about a paper on which we are a co-author, given our excitement for discovery no matter how busy we are. That is, for some of us we will prioritize papers, especially ones from mentees, such that a response can be expected within one week. This does not make us super heroes; it is simply a reflection of our excitement for science and respect for co-authors and mentees.

We urge authors, especially early career ones, not to give up when they encounter a tardy or unresponsive coauthor. You are not alone! Perhaps the worst outcome of dealing with a tardy or unresponsive co-author is to shelve a writing project entirely, such that the work never reaches the scientific community. Instead, consider our advice and also consult colleagues. Be respectful of the scientific process and recognize that reviews can take time, especially when dealing with early career writers with a tendency for verbosity. That said, co-authors (which are collaborators) also must realize that being tardy or unresponsive can have negative consequences. The author and co-authors alike play a role in this process. We also must be clear that in no way should the process we describe above be used to push through a paper in a manner to be deceitful. Nor do we advocate rushing through the pre-submission editing and polishing phases of manuscript preparation. And if the co-author has some form of expertise or input that is critical to a paper, it may be necessary to bring on an additional co-author or seek a "friendly review" from a colleague. After all, the publication process is about refinement of ideas and papers and should not suffer because of speed (Donaldson and Cooke 2014), either too fast or too slow!

\section{Acknowledgements}

Cooke is supported by the Canada Research Chairs program and Donaldson and Cooke are further supported by NSERC. We thank Trevor Branch for encouraging us to think about this topic.

\section{References}

Donaldson, M.R. and S.J. Cooke. 2014. Scientific publications: Moving beyond quality and quantity toward influence. BioScience 64: 12-13. CrossRef

Rowland, F. 2002. The peer-review process. Learned Publishing 15: 247-258. CrossRef

Schäfer, R.B., S.J. Cooke, R. Arlinghaus, N. Bonada, F. Brischoux, A.F. Casper, J.A. Catford, and V. Rolland. 2011. Early career researchers' perspectives on the current and future state of the scientific publication process in ecology. Freshwater Biology 56: 2405-2412. CrossRef 